\title{
Subwavelength Grating Waveguide Devices for Telecommunications Applications
}

\author{
Lawrence R. Chen, Senior Member, IEEE, Junjia Wang, Behnam Naghdi, \\ and Ivan Glesk, Senior Member, IEEE
}

(Invited Paper)

\begin{abstract}
Subwavelength grating (SWG) waveguides in siliconon-insulator are emerging as an enabling technology for implementing compact, high-performance photonic integrated devices and circuits for signal processing and sensing applications. We provide an overview of recent work on developing wavelength selective SWG waveguide filters based on Bragg gratings, ring resonators, and contra-directional couplers, as well as optical delay lines for applications in optical communications and microwave photonics. These components increase the SWG waveguide component toolbox and can be used to realize more complex photonic integrated circuits with enhanced or new functionality.
\end{abstract}

Index Terms-Subwavelength gratings, silicon photonics, integrated optics, Bragg gratings, ring resonators, contradirectional couplers, optical add-drop multiplexers, optical delay lines

\section{INTRODUCTION}

$I^{1}$ NTEGRATED photonic solutions are in demand for diverse applications ranging from data communications to instrumentation to sensing. Integrated optical devices in silicon, including those based on silicon-on-insulator (SOI) and silicon nitride ( $\mathrm{SiN}$ ), can leverage CMOS fabrication facilities. The use of silicon for realizing integrated optical components was proposed more than 30 years ago [1] and interest, research, and development in silicon photonics (SiP) in academia as well as industry started its incredible acceleration and growth about 15 years ago [2-4]. This interest continues today and over the years, there have been a number of impressive realizations and demonstrations of passive and active devices, as well as integrated subsystems, and deployment of commercial products from communications to biosensing (e.g., from Acacia Communications, Luxtera, and Genalyte, to name a few). SiP has led to transformative technologies for coherent optical communications and is a driving force behind the rapidly

Submitted 17 October 2018.

This work was supported in part by the Natural Sciences and Engineering Research Council of Canada (NSERC) NGON and Si-EPIC CREATE programs, an NSERC Strategic Project Grant, and the Royal Society International Exchanges Scheme 2012/R2

L. R. Chen and B. Naghdi are with the Department of Electrical and Computer Engineering, McGill University, Montreal, QC H3A 0E9 Canada (lawrence.chen@mcgill.ca, behnam.naghdi@mail.mcgill.ca). emerging field of integrated microwave photonics (MWP).

Among the various developments in SiP, one area that has started to attract increased interest is the use of subwavelength grating (SWG) structures, such as an SWG waveguide as shown in Fig. 1, for designing and implementing integrated optical components [5-7]. An SWG is based on a periodic arrangement of two different materials having a period that is much smaller than the wavelength of the light $[8,9]$. Light can propagate in the direction perpendicular to the subwavelength structure - referred to as crosswise operation —or parallel to the axis of the structure - referred to as lengthwise operation. The interest in SWG structures is the ability to perform refractive index engineering. A number of SWG components in SOI that use crosswise operation have been reported, especially highefficiency fiber-to-chip surface grating couplers [10-12].

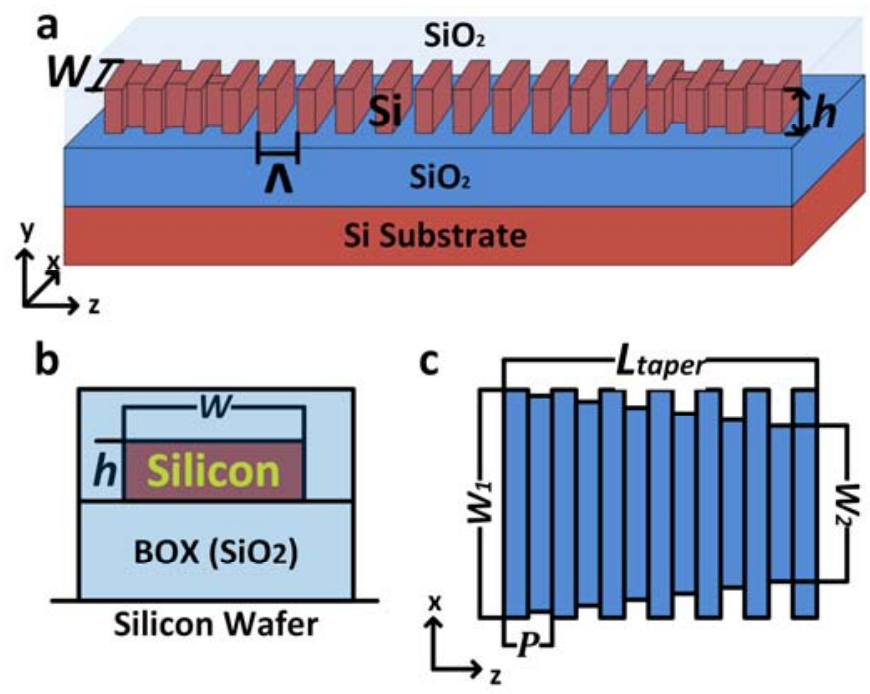

Fig. 1. (a) Schematic of SWG waveguide in SOI, (b) waveguide crosssection, and (c) SWG taper used to couple light from a conventional strip waveguide (or nanowire waveguide) to the SWG waveguide.

J. Wang was with the Department of Electrical and Computer Engineering, McGill University, Montreal, QC H3A OE9 Canada. He is now with the Cambridge Graphene Center, Department of Engineering, University of Cambridge, Cambridge CB3 0FA, UK (jw973@cam.ac.uk).

I. Glesk is with the Department of Electronic and Electrical Engineering, University of Strathclyde, Glasgow G1 1XW, UK (ivan.glesk@strath.ac.uk). 
Since the first demonstration of an optical waveguide with an SWG metamaterial core by Bock et al. [13], metamaterial SWG waveguides have attracted a strong research interest because of their unique potential to control light propagation. In particular, researchers have exploited lengthwise operation to implement 'simple' passive SWG waveguide-based devices such as couplers and beam splitters, both directional couplers (DCs), and multimode interference couplers [14,15]; mode transformers [16]; and polarization converters [17]. A key operating characteristic of these SWG waveguide-based devices compared to their traditional counterparts is the ultrabroadband operation (e.g., [15]). Low loss bends [18] and waveguide crossings [19] have also been demonstrated. These 'building blocks' are compatible and can be used to develop more complex integrated structures, photonic integrated circuits (PICs), and (sub-)systems. From a practical perspective, the majority of these devices can be obtained using a single etch, which simplifies fabrication and processing.

In this paper, we review recent progress on developing additional basic building blocks based on SWG waveguides in SOI for applications in optical communications and MWP, particularly optical filters and optical delay lines (ODLs). We describe how Bragg gratings (BGs) [20-23], microring resonators (MRRs) [20,21,24,25], contra-DCs [26-28], and optical add-drop multiplexers (OADMs) [29] can be implemented using SWG waveguide components, as well as how SWG waveguides enable index variable optical true time delay lines (OTTDLs) [30].

\section{BASIC BUILDING BLOCKS}

Fig. 1 illustrates the schematic of an SWG waveguide in SOI. The SWG is formed by a periodic arrangement of high refractive index material (i.e., Si) with a thickness $a$ that is embedded within a medium of lower refractive index (e.g., $\mathrm{SiO}_{2}$ ). SWGs are characterized by having a period $\Lambda$ that is sufficiently small compared to the wavelength of light. The duty cycle of the SWG is defined as $D=a / \Lambda$. An SWG waveguide is formed by defining finite transverse dimensions, e.g., a cross-section defined by a height $h$ and a width $w$, to the high refractive index material.

Light propagating in an SWG waveguide excites a combination of the Bloch modes of the periodic structure. Bloch waves are the natural modes of periodic media in the same way that plane waves are the natural modes of free space. The propagation of a Bloch mode through the SWG waveguide can be described by the following expression:

$$
E(x, y, z+\Lambda)=E_{B}(x, y, z) e^{-\gamma_{B} \Lambda}
$$

where $E_{B}(x, y, z)$ is the Bloch mode field distribution within a single period and $\gamma_{B}$ is its corresponding complex propagation constant that determines how the field repeats itself after propagating one period. In general, $\gamma_{B}=\alpha_{B}+j k_{B}=\alpha_{B}+$ $j(2 \pi / \lambda) n_{B}$ where $\alpha_{B}$ and $k_{B}$ are the attenuation and propagations constants (wave number), respectively, and $n_{B}$ is the effective index of the Bloch mode.

The carrier frequency of the light signal propagating in the SWG waveguide determines the operating regime and operation can be divided generally into the following three regimes [5-7].

Diffraction regime. This regime is a consequence of phasematched coupling between the radiation modes of the structure and its index guided modes. The gratings provide a phase factor of $q \times K$ along the propagation axis, here $z$, where $K=2 \pi / \lambda$ and $q$ is the diffraction order, and if the phase-matching condition $k_{i} \sin \left(\theta_{i} q\right)+q K=k_{B}$ is satisfied (where $\theta_{i}$ is the diffraction angle), the Bloch mode is leaky and the power of the launched light diffracts out of the waveguide core.

Reflection regime. Carrier frequencies below those associated with the diffraction regime may fall within the photonic bandgap, where the propagating light cannot be efficiently phase-matched to the radiation modes but rather successive reflections from the grating structure are able to add constructively giving rise to strong Bragg reflection. Thus, the incoming light is gradually attenuated and is reflected back to the input. In this regime, $k_{B}$ is a constant, $k_{B}=k_{B, B r a g g}=$ $\pi / \lambda$ and the corresponding Bragg wavelength is $\lambda=$ $2 n_{B, B r a g g} \Lambda$.

Subwavelength regime: At even lower frequencies, i.e., below the first photonic bandgap, diffraction orders at an angle $\theta$ will not be phase-matched with the guided mode and are not able to receive power. Similarly, successive back reflections cannot add constructively. Thus, both diffraction and reflection effects are suppressed. In this regime, the propagation constant $k_{B}$ increases monotonically with frequency, in a manner similar to a conventional optical waveguide. Interestingly, light is able to propagate through thousands of SWG discontinuities with no loss (theoretically speaking). The subwavelength regime is obtained when the period of the SWG is small enough so that the effective index of the Bloch mode falls below the condition for Bragg reflection, i.e., $n_{B}<n_{B, \text { Bragg }}$ or $\lambda>2 n_{B, B \text { ragg }} \Lambda$.

We are particularly interested in the subwavelength regime where the grating can be modeled as a conventional waveguide having the same transverse dimensions but a uniform core material with an equivalent effective refractive index, $n_{\text {eff }}$. This effective index depends on the duty cycle $D$ [5-9].
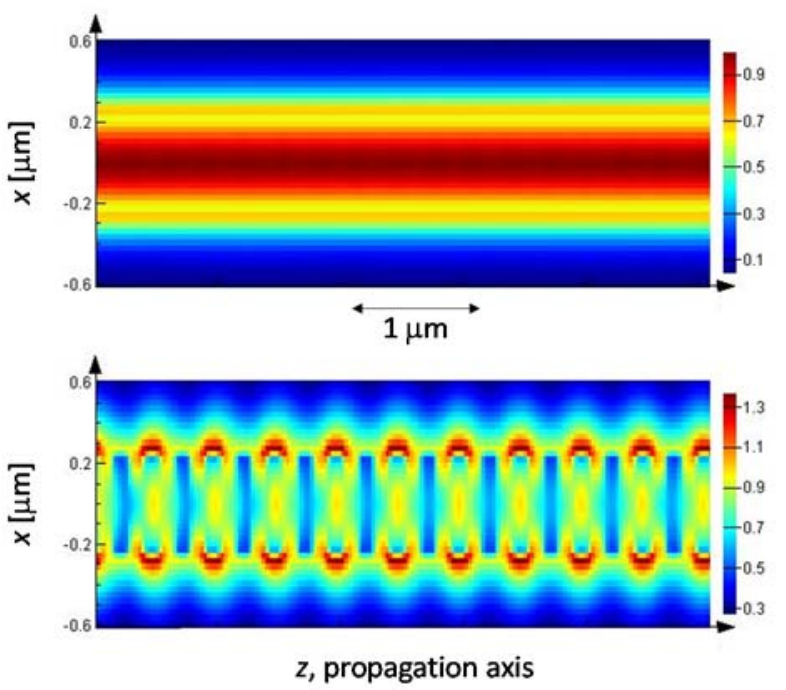

Fig. 2. Simulated $E$-field for a TE-like mode propagating in a strip waveguide and a Bloch mode propagating in an SWG waveguide in SOI. The waveguides have the same cross-section. 


\section{A. Waveguides and crossings}

We begin by examining propagation in a conventional strip waveguide and an SWG waveguide in SOI. The waveguides have the same transverse dimensions of $h=220 \mathrm{~nm} \times w=500$ $\mathrm{nm}$; the SWG waveguide has a period $\Lambda=300 \mathrm{~nm}$ and $D=50 \%$. The waveguides 'sit' on a buried oxide (BOX) layer and are covered by a cladding that is index-matched to the BOX layer. Fig. 2 shows the $E$-field centered at a carrier wavelength of $1550 \mathrm{~nm}$ propagating in a strip waveguide and an SWG waveguide in SOI calculated using a 2.5D FDTD simulator (Lumerical ${ }^{\mathrm{TM}}$ ); the Sellmeier relations are used to account for the frequency dependence of the refractive indices. We can clearly observe the difference between the TE-like mode propagating in the strip waveguide and the Bloch mode propagating in the SWG waveguide. Note in the latter the absence of coupling to radiation modes or to Bragg reflection.

In the first report of index guiding in an SWG waveguide in SOI, Bock et al. used a cross-section of $h=260 \mathrm{~nm} \times w=300$ $\mathrm{nm}, \Lambda=300 \mathrm{~nm}$, and $D=33 \%$ [13]. Although theoretical predictions call for no propagation loss in SWG waveguides, the measured propagation loss in the $\mathrm{C}$-band for TE modes was found to be $\sim 2.1-2.6 \mathrm{~dB} / \mathrm{cm}$ with a polarization dependent loss (PDL) of $0.5 \mathrm{~dB}$. As mentioned in [13], this loss is "remarkable that such low loss is achieved for light propagating over a $1 \mathrm{~cm}$ distance through more than 33,000 boundaries between highand low-refractive-index segments having an index contrast of $\Delta \mathrm{n} \sim 1.9$."

Recently, Gao et al. investigated the transmission of data signals in an SWG waveguide in SOI [31]. Their $2 \mathrm{~mm}$ long SWG waveguide was based on a cross-section of $h=220 \mathrm{~nm} \times$ $w=300 \mathrm{~nm}, \Lambda=300 \mathrm{~nm}$, and $D=50 \%$. The propagation loss of $4.2 \mathrm{~dB} / \mathrm{cm}$ was higher than that in [13] which the authors attributed to imperfections arising during fabrication, particularly surface roughness and variability in the size of the silicon segments. They transmitted 38.12 Gbaud OFDM 16QAM signals on 75 WDM channels with a BER $<4 \times 10^{-5}$ on each channel (below the FEC threshold). While similar performance was obtained for transmission through a conventional strip in SOI (with $h=220 \mathrm{~nm} \times w=400 \mathrm{~nm}$ ), the SWG waveguide provided an $8 \mathrm{~dB}$ improvement in launch power due to reduced nonlinear effects.

The suppression of diffraction effects in SWG waveguides make them suitable for implementing waveguide crossings. Bock et al. demonstrated crossings based on SWG waveguides with $h=260 \mathrm{~nm} \times w=300 \mathrm{~nm}$ and $h=220 \mathrm{~nm} \times w=400 \mathrm{~nm}$, both with $D=50 \%$ [18]. For TE modes, losses of 0.023 $\mathrm{dB} /$ crossing and $0.07 \mathrm{~dB} /$ crossing were obtained for $w=300$ $\mathrm{nm}$ and $400 \mathrm{~nm}$, respectively. While smaller values of $w$ resulted in lower loss/crossing, they induced greater PDL $(<$ $0.02 \mathrm{~dB}$ compared with $<0.005 \mathrm{~dB}$ ). Crosstalk for both SWG waveguide designs was $<40 \mathrm{~dB}$. In contrast, losses of 0.98 $\mathrm{dB} /$ crossing and a crosstalk of $15 \mathrm{~dB}$ were obtained with strip waveguides.

\section{B. Tapers and bends}

As shown in Fig. 2, the modes propagating in strip and SWG waveguides are substantially different. If a PIC comprises both strip and SWG waveguides, then some form of transition between the modes will be necessary to reduce excess loss whenever the two different types of waveguides are interfaced. An SWG taper converts a conventional mode propagating in a strip waveguide into a Bloch mode that propagates in an SWG waveguide $[18,19]$. SWG tapers are designed for adiabatic adjustment of one mode to the other and transitioning gradually from the SWG core refractive index to that of the strip waveguide. The SWG taper structure is shown in Fig. 1(c). It is based on a periodic grating-like structure with period $P$ and where the waveguide width is varied linearly from $W_{1}$ to $W_{2}$ over a length $L_{\text {taper }}$.

Donzella et al. investigated the input and output mode mismatch loss as well as the total taper loss for SWG waveguides as a function of $D$ [19]. Taper losses were lower for larger values of $D$ as well as for TM modes. Generally speaking, it is possible to tailor the design of the taper to minimize the loss due to mode mismatch between a strip and SWG waveguide and a taper loss of $0.8 \mathrm{~dB}$ can be achieved. It should be mentioned that tapers were used in the SWG waveguides described in Section 2A; note that the losses reported exclude the taper/mode mismatch losses.

In addition to crossings, waveguide bends are essential for designing more complex PICs, e.g., for routing, as well as for developing ring-based structures (to be discussed in Section 3B). Donzella et al. also performed a detailed analysis of SWG waveguide bends [19]. A $90^{\circ}$ bending loss of $\sim 1.5 \mathrm{~dB}$ was demonstrated for a bend radius of $10 \mu \mathrm{m}$; this loss was reduced to $\sim 1 \mathrm{~dB}$ when the bend radius was increased to $30 \mu \mathrm{m}$. Moreover, lower losses can be supported using higher values of $D$. As demonstrated by Wang et al., using trapezoidal, rather than rectangular, silicon segments in the SWG waveguide bends should reduce losses even further [32].

\section{SWG WAVEGUIDE-BASED FILTERS}

In this section, we discuss the design and characteristics of optical filters based on SWG waveguides. For fabrication processes available from typical silicon photonic multi-project wafer runs, our devices are based on $h=220 \mathrm{~nm}$; the silicon layer sits on top of a $3 \mu \mathrm{m} \mathrm{BOX}$ and is covered by a $2 \mu \mathrm{m}$ cladding that is index-matched to the BOX, see Fig. 1(b). Our devices use tapers between strip and SWG waveguides. Finally, all our devices use focused vertical grating couplers (VGCs) for input and output coupling to optical fiber [33]. The VGCs are optimized for TE mode operation; as such, all device characteristics are reported for one polarization only (characteristics on PDL are not available). The VGCs span typically the C-band and have a loss of $7.5 \mathrm{~dB} /$ coupler. All our devices are fabricated using ebeam lithography and a single etch.

\section{A. Bragg gratings}

BGs can be realized by introducing a periodic variation along the direction of propagation in the waveguide. This variation can come in the form of physical corrugations, variations in the waveuigde geometry, or variations in the refractive index, e.g., 
through photoinduced effects.

BGs in conventional strip waveguides in SOI have been demonstrated. It is also possible to obtain Bragg reflection in an SWG waveguide. In particular, we can interleave two SWG waveguides having differing duty cycles to induce a longitudinal periodic variation in the effective refractive index $[20,22]$. Note that this SWG BG effectively involves two 'types' of SWGs: one is used to form the waveguide and the period is set to operate outside of the bandgap, i.e., the subwavelength regimes that supports low loss propagation, and the second has a larger period for Bragg reflection.

Fig. 3 depicts the schematic of an SWG BG that is formed by interleaving two SWG waveguides with duty cycles $D_{1}=$ $\left(a_{1} / \Lambda_{1}\right)$ and $D_{2}=\left(a_{2} / \Lambda_{2}\right)$. The period of the SWG BG is $\Lambda_{1}+$ $\Lambda_{2}$. We fabricated SWG BGs in SOI having a waveguide crosssection of $h=220 \mathrm{~nm} \times w=500 \mathrm{~nm}$ and periods $\Lambda_{1}=\Lambda_{2}=280$ $\mathrm{nm}$ [22]. The SWG BGs comprise 2,000 periods giving a total length of $1.12 \mathrm{~mm}$. We use a Y-branch based on strip waveguides to extract the reflection response. The strip and SWG waveguides are connected using an SWG taper with a period $P=200 \mathrm{~nm}$ and where the waveguide width is reduced from $W_{1}=500 \mathrm{~nm}$ to $W_{2}=200 \mathrm{~nm}$ over a length $L_{\text {taper }}=15 \mu \mathrm{m}$. All VGCs which provide input coupling as well as extraction of the reflection and transmission responses are placed on the same end of the device with a pitch of $127 \mu \mathrm{m}$ to match the fiber-to-fiber spacing of a fiber ribbon array. The device occupies a footprint of $1.18 \mathrm{~mm} \times 254 \mu \mathrm{m}$.
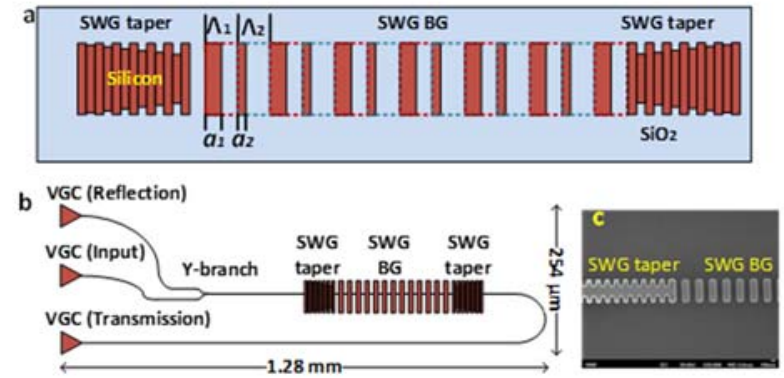

Fig. 3. SWG BG: (a) schematic showing two interleaved SWG waveguides as well as SWG tapers, (b) layout of fabricated device (dimensions of the SWG components are exaggerated for illustrative purposes), and (c) SEM of the SWG BG prior to depositing the oxide cladding.

In Fig. 4, we show the transmission response of an SWG waveguide as well as that of an SWG BG where $D_{1}=50 \%$ and $D_{2}=48 \%$. The transmission through the SWG BG is different due to the spectral response of the Y-branch, but more importantly, there is a clear rejection peak at $1546.8 \mathrm{~nm}$ due to Bragg reflection. From the reflection response, the $3 \mathrm{~dB}$ bandwidth is $0.5 \mathrm{~nm}$ and from the transmission loss at the resonant wavelength, we estimate the peak reflectivity to be $90.4 \%$. Changing the duty cycles of the interleaved SWG waveguides allows for control over the Bragg resonance. For example, when we use $D_{2}=44 \%$ in the SWG BG, the reflection peak is centered at $1533.2 \mathrm{~nm}$. The $3 \mathrm{~dB}$ bandwidth is similar $(0.5 \mathrm{~nm})$ as is the peak reflectivity $(94.4 \%)$. Note that it is possible to obtain narrowband reflection from Bragg gratings (e.g., patterned on the top surface or with sidewall corrugations) on ridge waveguides in SOI $[34,35]$.

Recently, Čtyrokýi et al. investigated alternate implementations of the SWG BG [23]. These include, amongst others, varying the longitudinal and transverse dimensions of one of the two interleaved SWG waveguides, or introducing loading segments at different locations along the length of the SWG waveguide. The additional degrees of freedom associated with the use of loading segments, e.g., through control of the position and size, provide further control of the spectral response of the SWG BG. In particular, it is possible to obtain apodized responses as well as Fabry-Pérot like structures. This increases the type of filtering operations that are possible using SWG BG structures.
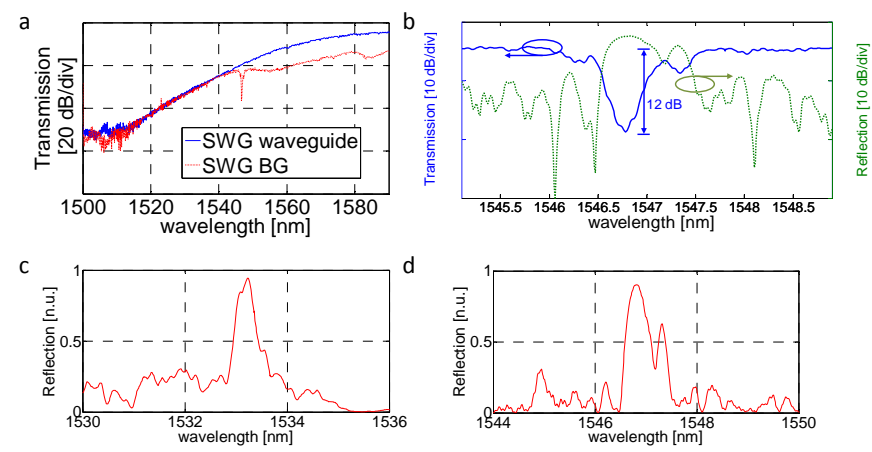

Fig. 4. Spectral responses of SWG BGs: (a) comparing the transmission spectrum of an SWG BG and a simple SWG waveguide, (b) SWG BG transmission (blue) and reflection (green) responses about the resonant peak at $1546.8 \mathrm{~nm}$, and (c) illustrating tuning of the Bragg resonance by varying the duty cycle: reflection response when $D_{1}=50 \%$ and $D_{2}=44 \%$ (left) and $D_{1}=$ $50 \%$ and $D_{2}=48 \%$ (right).

\section{B. Ring resonators}

MRRs provide high wavelength selectivity and can be used to develop compact integrated optical filters. In addition to filtering, MRRs have been used in modulators, detectors, and for slow light/tunable optical delay structures. MRRs have been demonstrated in SiP, including SOI and SiN. Since the properties of SWG waveguides can be readily controlled and low loss SWG waveguide bends can be obtained, it is also possible to implement MRRs based on SWG waveguides.
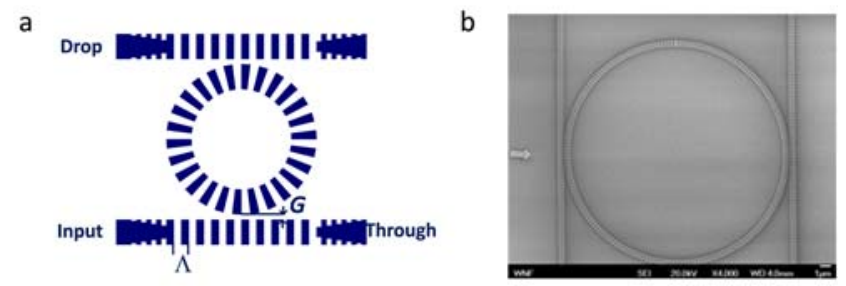

Fig. 5. (a) Schematic or SWG waveguide MRR and (b) SEM of fabricated device before top oxide cladding deposition.

Recently, we demonstrated MRRs based on SWG waveguides in SOI with a top oxide cladding $[20,21]$ while Donzella et al. reported similar structures but without any upper cladding [24,25]. Fig. 5(a) shows a conventional add-drop MRR configuration comprising two SWG bus waveguides and 
an SWG ring waveguide. We consider SWG waveguides with a cross-section $h=220 \mathrm{~nm} \times w=500 \mathrm{~nm}, \Lambda=300 \mathrm{~nm}$, and $D$ $=50 \%$. Note that in the ring, the period of the SWG waveguide is not exactly $300 \mathrm{~nm}$ since the exact value of the period depends on the position of the silicon segments as the ring is sliced by degrees. The bus and ring waveguides are coupled via directional coupling with a gap separation (separation between waveguides and not the center-to-center separation) $G=140$ $\mathrm{nm}$. We use SWG tapers which are similar in design to those used for the BGs. Fig. 5(b) shows an SEM of the fabricated SWG waveguide MRR prior to deposition of the top oxide cladding.

Fig. 6 shows the through and drop responses of SWG waveguide MRRs with ring radii of $15 \mu \mathrm{m}, 20 \mu \mathrm{m}$, and $25 \mu \mathrm{m}$; as expected, devices having smaller ring radii have larger free spectral range (FSR). For our devices, the $Q$-factor (defined as $\lambda_{0} / \Delta \lambda$ where $\lambda_{0}$ is the central wavelength of a resonance and $\Delta \lambda$ is the corresponding $3 \mathrm{~dB}$ bandwidth) for MRRs with larger ring radii is higher $(1,280$ for a ring radius of $25 \mu \mathrm{m}$ compared 356 for a ring radius of $15 \mu \mathrm{m}$ ). On the other hand, they also exhibit lower extinction ratio (ER) measured at the through response of the resonance peaks (an average of $\sim 6 \mathrm{~dB}$ for a ring radius of $25 \mu \mathrm{m}$ compared to $15 \mathrm{~dB}$ for a ring radius of $15 \mu \mathrm{m}$ ).
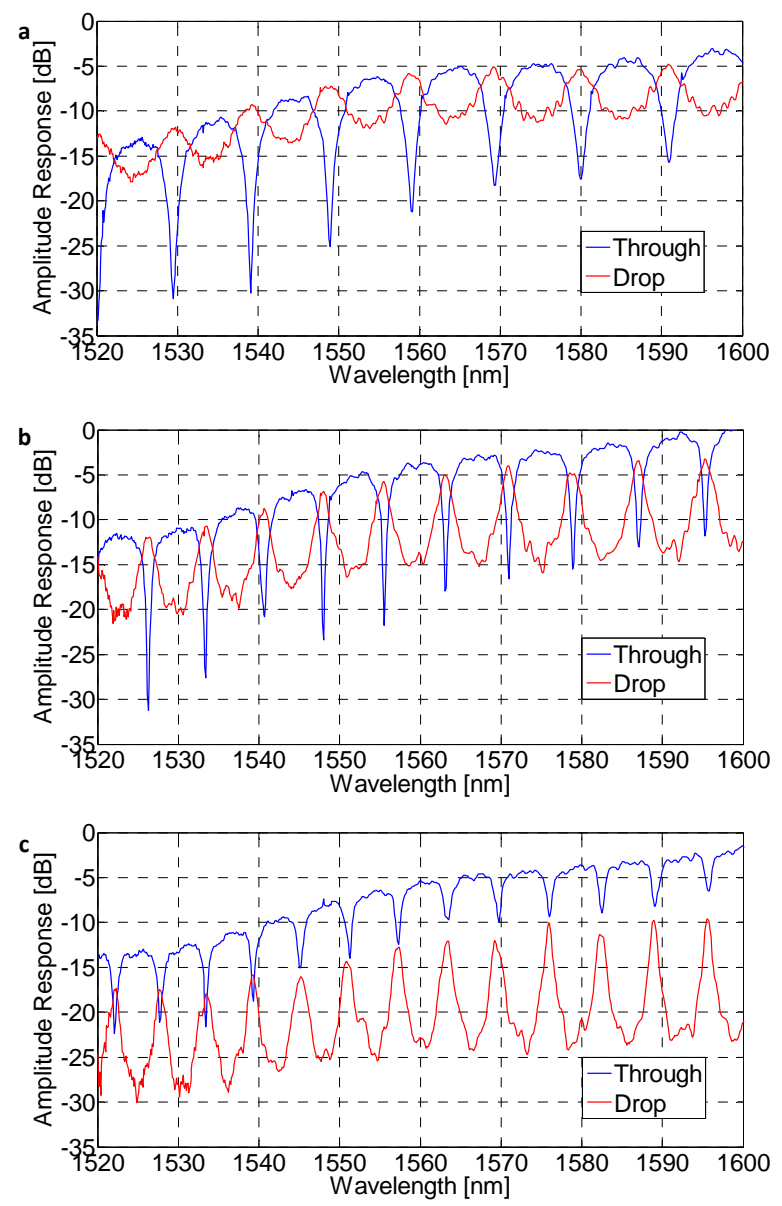

Fig. 6. SWG waveguide MRR: measured through and drop responses for devices with a ring radius of (a) $15 \mu \mathrm{m}$, (b) $20 \mu \mathrm{m}$, and (c) $25 \mu \mathrm{m}$. Measurement resolution: $10 \mathrm{pm}$.
A racetrack design is sometimes more preferable over the conventional ring since it is possible to exercise increased control over the coupling efficiency due to the longer coupling length between the bus and resonator waveguides. In particular, by controlling the coupling length, it can be easier to obtain a desired coupling condition: under-coupled, critically coupled, or over-coupled. Figs. 7(a) and (b) illustrate the layout and schematic of the racetrack resonator based on SWG waveguides. The length of the directional coupling section, $L$, and the gap, $G$, determine the coupling efficiency and hence operating condition. The curved section of the resonator has a radius $r$ and the total perimeter of the racetrack is $2 \pi r+2 L$.

We fabricated a racetrack resonator with $r=20 \mu \mathrm{m}, L=$ $40 \mu \mathrm{m}$, and $G=100 \mathrm{~nm}$ (the SWG waveguide parameters are the same as those for the MRRs described earlier) [21]. The measured through and drop responses are shown in Fig. 7(c). The ER, which is as high as $33 \mathrm{~dB}$, is higher than that of the conventional MRRs shown in Fig. 6 , and the $3 \mathrm{~dB}$ bandwidth is $1 \mathrm{~nm}$.

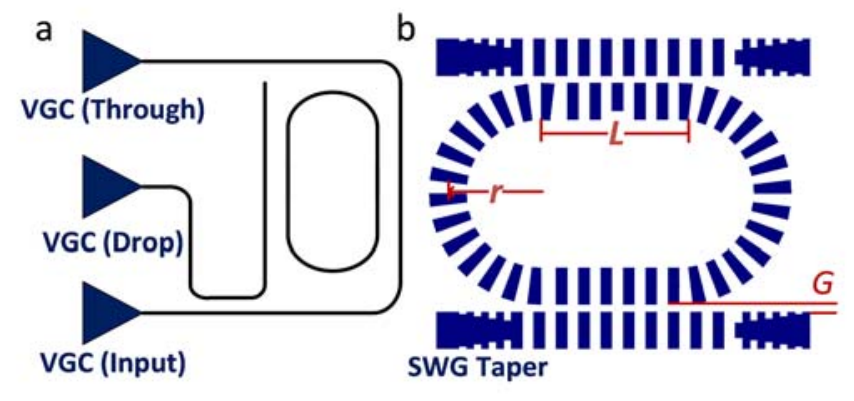

C

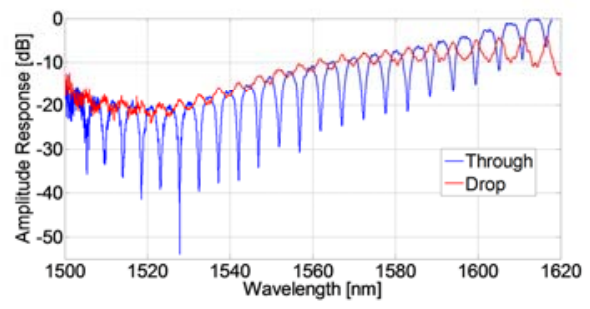

d

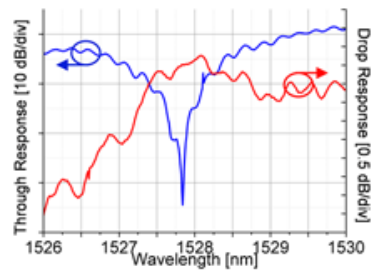

Fig. 7. SWG racetrack resonator: (a) layout and (b) schematic. (c) Measured through and drop responses of a fabricated device and (d) zoom of the resonance at $1527.85 \mathrm{~nm}$.

The total resonator losses are important for determining the $Q$-factor and ER. Factors which contribute to the resonator losses include SWG waveguide loss and bending loss. Although the optical mode in SWG waveguides is delocalized, thereby reducing interaction of the electric field with surface scattering sources, sidewall roughness, arising from the fabrication process, will contribute to loss and is an issue that needs to be carefully examined. The SWG waveguide bending 
loss depends on the duty cycle $D$. Donzella et al. also investigated in detail the characteristics of SWG waveguide MRRs and found the following [24]. Rings based on SWG waveguides with a smaller duty cycle have a higher $Q$-factor and ER for larger radii (e.g., $\geq 30 \mu \mathrm{m})$. This is due to the smaller loss associated with a smaller duty cycle (e.g., for $D=$ $70 \%$ compared to $80 \%$ ). On the other hand, rings based on SWG waveguides with a larger duty cycle have a higher $Q$ factor and ER for small radii (e.g., $10 \mu \mathrm{m}$ ). This is since SWG waveguides with lower duty cycle and small bend radii have higher mode mismatch (and hence higher total bending loss).

In our fabricated MRRs with a top oxide cladding, we obtained modest $Q$-factors of several hundred to $\sim 1,200$ and ERs ranging from a few $\mathrm{dB}$ up to $20 \mathrm{~dB}$ with a top oxide cladding, depending on ring radius. Donzella et al. achieved higher values [24,25], e.g., $Q$-factors up to 6,000 with an air cladding or 4,000 with a fluidic cladding, and ERs ranging from a few $\mathrm{dB}$ to $35 \mathrm{~dB}$.

Since the Bloch mode exists between the silicon segments, MRRs based on SWG waveguides improve the sensitivity of index sensors. For example, without a top oxide cladding (i.e., an air cladding), sensitivities exceeding $383 \mathrm{~nm} / \mathrm{RIU}$ in water and $270 \mathrm{~nm} / \mathrm{RIU}$ in air, which are greater than those obtained with rings resonators based on conventional silicon slot and strip waveguides, were obtained [24].

The various results on SWG waveguide MRRs obtained to date are promising. Further work must address the issue of losses further to enhance the $Q$-factor and/or ER so that they have comparable performance to resonators based on conventional strip waveguides. It may also be possible to envision coupled resonators to tailor further the spectral responses for specific filtering and signal processing applications.

\section{Wideband filters}

Although BGs and MRRs are popular for designing optical filters, they present several shortcomings. BGs typically operate in reflection mode and as such, there is a need for optical circulators or some form of splitter/coupler (e.g., a $3 \mathrm{~dB}$ coupler or a Y-branch) which either increases complexity for integration or introduces additional loss. On the other hand, MRRs have a periodic response which may be undesirable for certain applications. Increasing the FSR of MRR filters requires the use of very small rings which may not be possible or is constrained by the amount of loss associated with tight bends. Moreover, even with higher-order MRR designs, the bandwidths are not always suitable for broadband filtering applications such as band splitting and band switching.

Grating-assisted contra- DCs can provide wideband filtering characteristics and take advantage of design flexibility similar to BGs [36]. Moreover, they are intrinsically four-port devices and thus, they can be employed readily in add-drop configurations. A grating-assisted contra-DC comprises two asymmetric waveguides that are closely spaced and some form of periodic refractive index perturbation along the direction of propagation in the waveguides, e.g., a grating. Recently, we showed an alternate implementation in which an SWG waveguide placed in close proximity to a strip waveguide can play the dual role of providing asymmetry as well as the periodic perturbation for contra-DC [26]. Due to the large optical phase mismatch between the SWG and strip waveguides in SOI, undesired co-DC can also be suppressed. The strong index variation in the SWG results in a strong coupling condition to transfer power using short coupling lengths.

Figs. 8(a) and (b) illustrate the schematic and layout of the SWG waveguide-based contra-DC. A strip waveguide is brought gradually into close contact with an SWG waveguide where they are separated by a distance $G$ and interact over a coupling length $L_{C}$. As with the other devices described previously, the cross-sections of the SWG and strip waveguides are $h=220 \mathrm{~nm} \times w=500 \mathrm{~nm}$; the SWG tapers have $P=250$ $\mathrm{nm}, W_{1}=500 \mathrm{~nm}, W_{2}=150 \mathrm{~nm}$, and $L_{\text {taper }}=15 \mu \mathrm{m}$. We consider an SWG period of $\Lambda=378 \mathrm{~nm}$ and $D=50 \%$. Fig. $8(\mathrm{c})$ shows the measured through and drop responses for an SWG contra-DC where $G=150 \mathrm{~nm}$ and $L_{C}=400 \mu \mathrm{m}$. The contraDC exhibits a bandwidth of $16 \mathrm{~nm}$ and an extinction ratio in excess of $35 \mathrm{~dB}$. As described previously, the large asymmetry in the effective refractive indices between the SWG and strip waveguides results in a large spectral separation between the resonant (drop) wavelength at $\lambda_{C}$ and unwanted intrawaveguide back reflections at $\lambda_{R 1}$ and $\lambda_{R 2}$. This is highlighted in Fig. 8(d), which plots the simulated add and drop responses for an SWG contra-DC with $\Lambda=378 \mathrm{~nm}, G=200 \mathrm{~nm}$, and $L_{C}$ $=100 \mu \mathrm{m}$ : there is a single operating band spanning $200 \mathrm{~nm}$, which exceeds that of previously demonstrated grating-assisted contra-DCs in SOI.

a
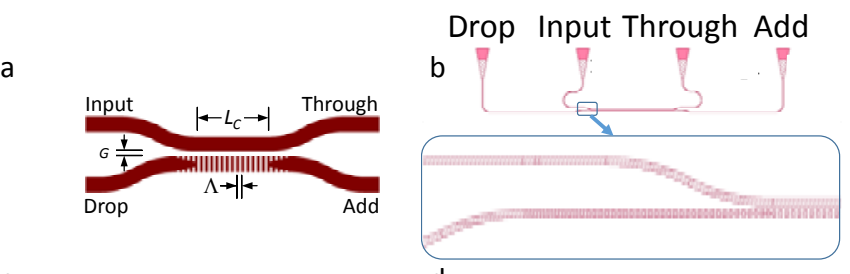

C
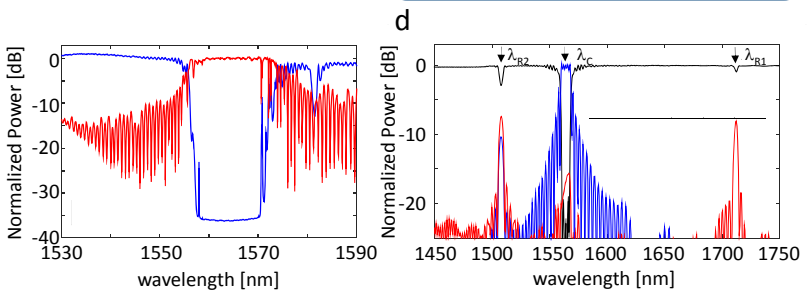

Fig. 8. SWG waveguide-based contra-DC: (a) schematic, (b) layout of a device (c) measured drop (red) and through (blue) responses for $\Lambda=378 \mathrm{~nm}$, $D=50 \%, G=150 \mathrm{~nm}$, and $L_{C}=400 \mu \mathrm{m}$, and (d) 3D FDTD simulation of response for $\Lambda=378 \mathrm{~nm}, D=50 \%, G=200 \mathrm{~nm}$, and $L_{C}=100 \mu \mathrm{m}$.

A contra-DC with a constant gap over the coupling length will have a spectral response that contains significant sidelobes; these make the device unsuitable for providing wavelength selectivity in OADMs. It is well known that apodization can be used to reduce such sidelobes. In [27], Liu et al. vary the gap distance from $900 \mathrm{~nm}$ to $200 \mathrm{~nm}$ for a $100 \mu \mathrm{m}$ coupler following a Gaussian function. In this case, a suppression of $27 \mathrm{~dB}$ in the sidelobes was obtained. However, the rejection ratio of the 
through port is only $\sim 5 \mathrm{~dB}$ within its stop band, and the pass band of the drop port has a $2.5 \mathrm{~dB}$ insertion loss over its $3 \mathrm{~dB}$ bandwidth. As such, while the device can be used as a two-port bandpass filter, it is not suited for providing add-drop functionality. To address this issue, we followed a design based on stronger power coupling using a narrower gap distance while adjusting the coupling length to obtain a compromise between sidelobe suppression and stop band/pass band extinction [28].

Fig. 9(a) portrays the schematic of our apodized SWG contra-DC design. A strip waveguide is brought gradually to a gap distance, $G_{\max }$, of a straight SWG waveguide to form the coupler waist over a coupling length $L_{\mathrm{C}}$. In contrast to [27], we then taper the gap using a raised cosine profile (for $0<z<$ $\left.L_{C}\right)$ :

$$
G(z)=G_{\max }-\frac{1}{2}\left(G_{\max }-G_{\min }\right)\left[1-\cos \left(\frac{2 \pi z}{L_{C}}\right)\right]
$$

The minimum gap at the center of the device, i.e., at $z=L_{C} / 2$, is $G_{\text {min }}$. Fig. 9(b) shows the measured spectral response of a fabricated contra-DC where $G_{\min }=150 \mathrm{~nm}, G_{\max }=450 \mathrm{~nm}$, $L_{C}=100 \mu \mathrm{m}$, and $\Lambda=378 \mathrm{~nm}$. The device has $19 \mathrm{~dB}$ suppression of the main sidelobe and $14 \mathrm{~dB}$ pass band/stop band extinction ratio with a $3 \mathrm{~dB}$ bandwidth of $12 \mathrm{~nm}$. For comparison, a device having the same gap variation but double the coupling length is also shown. In this case, the ER increases to $33 \mathrm{~dB}$ while the $3 \mathrm{~dB}$ bandwidth is almost the same.
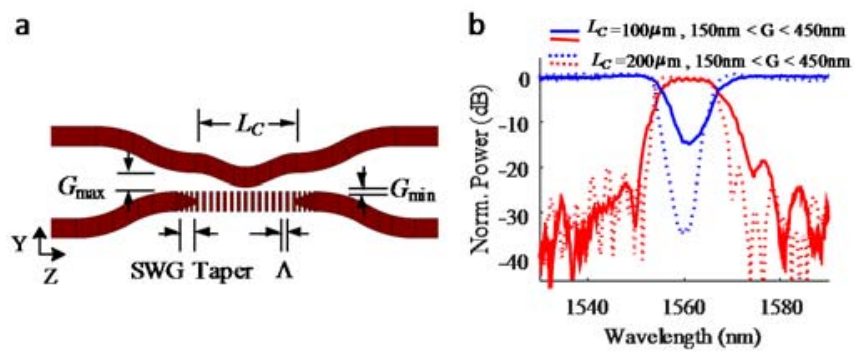

Fig. 9. Apodized SWG waveguide-based contra-DC: (a) schematic and (b) measured drop port (red) and through port (blue) spectra for devices with $G_{\min }$ $=150 \mathrm{~nm}, G_{\max }=450 \mathrm{~nm}$, and $L_{C}=100 \mu \mathrm{m}$ (solid lines) and $200 \mu \mathrm{m}$ (dotted lines).

From the perspective of coupled mode theory, it should not matter whether one tapers the gap distance based on curving the strip waveguide (as we have done) or the SWG waveguide (as in [27]). Our simulations verified this expectation showing no significant difference in spectral response as long as the gap profile remained the same. However, it may be simpler to employ a curved strip waveguide compared to a curved SWG waveguide. Nevertheless, the design was basically aimed at providing a compromise between sidelobe suppression and ER.
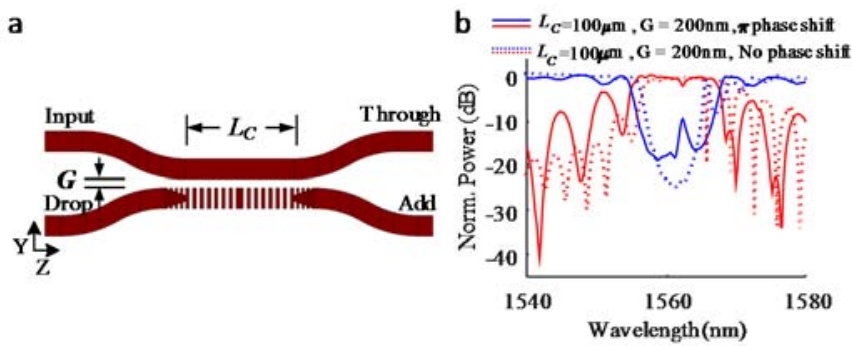

Fig. 10. Phase-shifted SWG waveguide-based contra-DC: (a) schematic and (b) measured drop port (red) and through port (blue) spectra for a device without (dotted lines) and with a $\pi$ phase shift (solid lines) at the center of the SWG waveguide.

Separating a pair of periodic structures by a quarter wavelength phase offset creates a single mode cavity and introduces a narrow band resonant transmission within the wide stop band of the periodic structures [37]. Accordingly, we have also investigated the possibility of creating a resonant filter by inserting a $\pi$ phase shift into the center of the SWG waveguide in the contra-DCs as shown in Fig. 10. Fig. 10 also compares the response of an SWG waveguide-based contra-DC without and with a $\pi$ phase shift. Here, we use a uniform gap of $G=$ $200 \mathrm{~nm}$ and $L_{C}=100 \mu \mathrm{m}$ (as before, $\Lambda=378 \mathrm{~nm}$ ). The phaseshifted structure clearly shows a transmission resonance within its stop band.

Recently, Charron et al. demonstrated an SWG waveguidebased contra-DC design that supports ultrabroadband operation [38]. The SWG waveguide consists of alternating subwavelength elements that are laterally displaced. The SWG waveguide is then brought into close contact to a strip waveguide in which sidewall corrugations are introduced to create a BG. The SWG elements and sidewall corrugations in the strip waveguide are in phase. This structure has a gap between the SWG and the strip waveguides which changes in a periodic manner to enable spatial modulation of the coupling. With this structure, a stopband of up to $33.4 \mathrm{~nm}$ was demonstrated, which is the largest reported to date. Moreover, by inserting a $\pi$ phase shift in the center of the BG, a transmission resonance with a bandwidth of $0.8 \mathrm{~nm}$ was obtained in the stop band.

Finally, $\mathrm{R}$ et al. demonstrated an ultra-broadband filter/switch by integrating SWG waveguides in a $2 \times 2$ MachZehnder interferometer (MZI) [39]. The SWG waveguides and device are designed so that successive stop bands and pass bands in the drop or cross/bar ports of the MZI. A band edge of $1565 \mathrm{~nm}$ was obtained with a stop band extinction of $35 \mathrm{~dB}$.

\section{Optical add-drop multiplexers}

Shi et al. demonstrated that it is possible to design an OADM by cascading multiple grating-assisted contra-DCs operating at different wavelengths [40]. We adopted a similar strategy whereby multiple SWG waveguide-based contra-DCs were cascaded in order to implement a multi-channel OADM [29]. Fig. 11 illustrates the layout of a four-channel, 10-port device: each channel has its own input and output port and there is a common input and through port. Light is coupled into and out of the device using VGCs, which are all aligned on the same side of the chip with a spacing of $127 \mu \mathrm{m}$. 


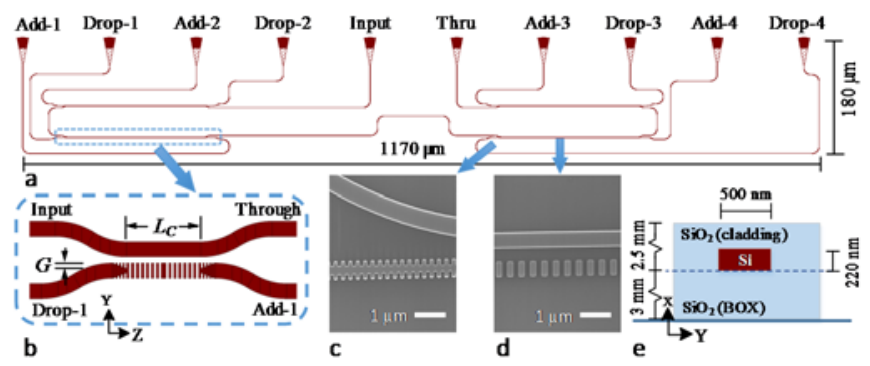

Fig. 11. (a) Layout of a four-channel OADM using SWG waveguide-based contra-DCs in SOI, (b) schematic top view of the gap-apodized contra-DCs, $(\mathrm{c}, \mathrm{d})$ SEM images of the mode-transition and coupler regions, and (e) waveguide cross-section.

The cascaded contra-DCs are based on the gap-apodized structure described in Section 3C with the following parameters: $G_{\min }=200 \mathrm{~nm}, G_{\max }=400 \mathrm{~nm}$, and $L_{C}=200 \mu \mathrm{m}$. The SWG periods are $\Lambda=370 \mathrm{mn}, 374 \mathrm{~nm}, 378 \mathrm{~nm}$, and 382 $\mathrm{nm}$ for channels 1 to 4 and a duty cycle of $D=50 \%$ is used. The same waveguide cross-section and SWG tapers described in Section $3 \mathrm{C}$ are also used. The total chip dimensions including the VGCs for input and output coupling are $1170 \mu \mathrm{m}$ $\times 180 \mu \mathrm{m}$ while the footprint of each SWG waveguide-based contra-DC is $250 \mu \mathrm{m} \times 20 \mu \mathrm{m}$.

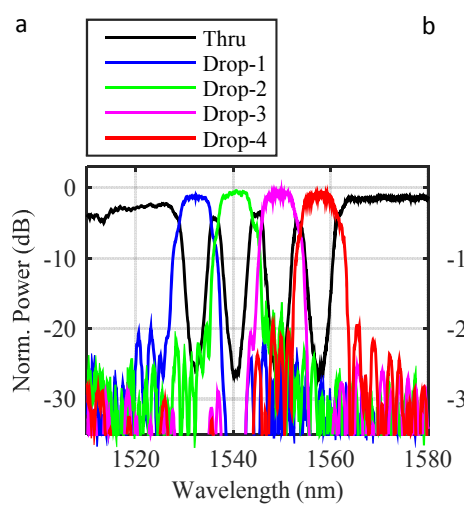

b
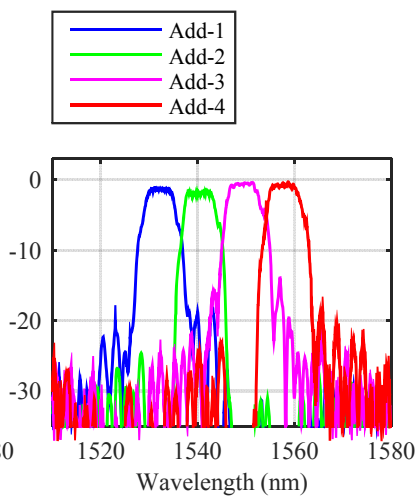

Fig. 12. Spectral response of four-channel OADM: (a) output at the drop ports and through port when light is launched into the input port and (b) output at the through port when light is launched into the different add ports. Wavelength resolution: $50 \mathrm{pm}$.

The spectral response of the OADM is shown in Fig. 12, where all results are normalized to the back-to-back insertion loss of a test pair of VGCs having a central loss of $\sim 15 \mathrm{~dB}$. The central wavelengths of the channels are $1532.3 \mathrm{~nm}, 1540.6 \mathrm{~nm}$, $1549.4 \mathrm{~nm}$, and $1557.8 \mathrm{~nm}$ with corresponding $3 \mathrm{~dB}$ bandwidths of $\sim 6.7 \mathrm{~nm}$. The transmission isolation at the center of each channel is $\sim 25 \mathrm{~dB}$.

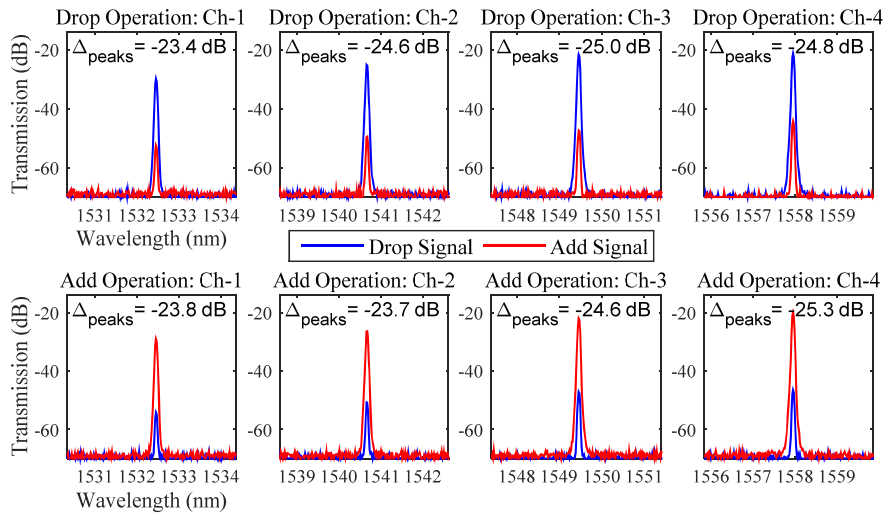

Fig. 13. Interferometric crosstalk measured at the center wavelength of each channel at the drop and through ports for drop and add operation. The blue (red) traces represent the drop (add) signals and the difference in the peaks of the plots denoted by $\Delta_{\text {peaks }}$ correspond to the levels of interferometric crosstalk.

One important characteristic of OADMs is interferometric (intrachannel) crosstalk, which occurs when the drop and add signals are at the same wavelength within a channel and the OADM does not have infinite isolation [41]. To evaluate interferometric crosstalk for a dropped signal (i.e., at the drop port), we compared the spectral output when a signal at a given channel wavelength is launched only at the input port to that when the signal at the same wavelength is launched at the add port. Similarly, by comparing the output at the through port under similar excitation conditions, we can determine the interferometric crosstalk for an add signal (i.e., at the through port). The results are summarized in Fig. 13 for the case where the signal is tuned to the center wavelength of each channel. The differences in the peaks of the traces, denoted by $\Delta_{\text {peaks, }}$, indicate the level of the interferometric crosstalk, which range from $-25.3 \mathrm{~dB}$ (for channel 4) to $-23.4 \mathrm{~dB}$ (for channel 1). These are comparable to the values of the transmission isolation. We assess further the impact of interferometric crosstalk with BER measurements. In particular, we consider $10 \mathrm{Gbit} / \mathrm{s}$ NRZ-OOK signals with a $2^{31}-1$ pseudo random bit sequence (PRBS) that are dropped or added from the OADM with and without interferometric crosstalk. The results, which are highlighted in Fig. 14, show that power penalties of $<2.0$ and $<2.8 \mathrm{~dB}$ are incurred for the drop and add operation, respectively.

A stronger gap apodization can provide larger suppression of the sidelobes. BER measurements, however, show negligible power penalty for interchannel crosstalk. Thus, using a stronger gap apodization to reduce the sidelobes will not improve further BER performance with regards to interchannel crosstalk; rather it will come at the expense of a weaker coupling regime, and consequently, lower transmission isolation, and thus increased interferometric crosstalk. 

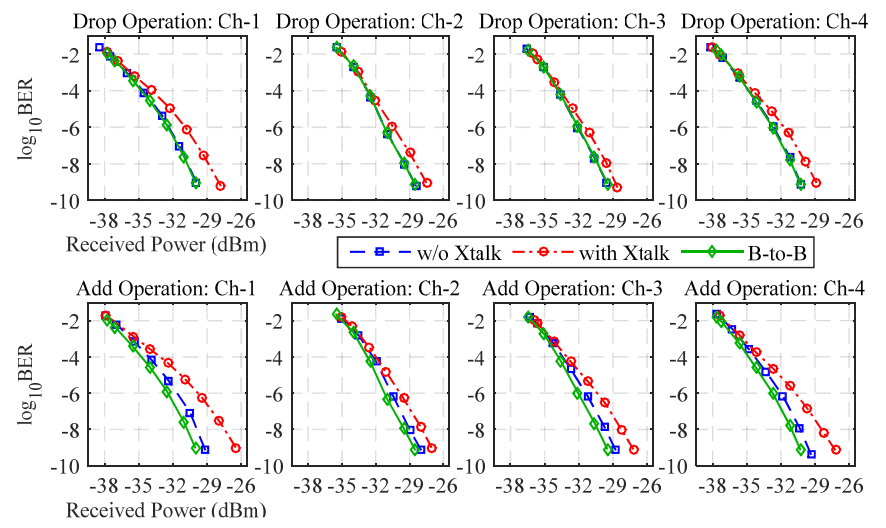

Fig. 14. BER measurements with (dot-dashed red) and without (dashed blue) the interferometric crosstalk compared to the back-to-back case (solid green) for both the drop and add operation for a $10 \mathrm{Gbit} / \mathrm{s} \mathrm{NRZ-OOK}$ data signal tuned to the center wavelength of each channel.

\section{SWG-Waveguide Based Delay Lines}

An ODL is an important building block for realizing various signal processing functions in optical communications [42] and MWP [43]. Large delay, small incremental delay steps (discrete tunability) or continuously tunable delay, broad operating bandwidth, and low loss are important features of ODLs; the specific values or combination of features required will be application-specific and generally, not all features will be required simultaneously.

Optical delays can be implemented with or without gain. The latter, passive approach can be further divided in two main categories: (1) length variable delay lines $[44,45]$ where the propagation length $(L)$ of the delay element is varied, i.e., the delay $\Delta t$ is set by $L / v_{\mathrm{g}}$ with $v_{g}$ being the propagation group velocity) and (2) variable propagation velocity delay lines [46] where $v_{g}$ is varied (in some implementations, this is also known as a wavelength variable delay line). These two approaches involve a fixed length of free space, fiber, or waveguide as the delay medium, or some form of resonance enhancement in which the physical path of the delay medium is enhanced through a cavity or by exploiting resonances where dispersion can be large. There is a well-known trade-off between the operating bandwidth and the size of resonance enhancement, and correspondingly the amount of optical delay [47]. In the discussion that follows, we focus on ODLs that are not based on resonance enhancements.

Wavelength variable ODLs have been used to implement sampled delay lines for MWP filtering. However, some applications require the time delays to be obtained without changes in the wavelength of the optical signal. An ODL that provides time delays for pulses or signals at the same optical carrier is one form of an optical true time delay line (OTTDL). The conventional means for implementing such an OTTDL uses an array of waveguides of different lengths whereby the incremental length between consecutive waveguides in the array defines the corresponding differential (or incremental) delay.
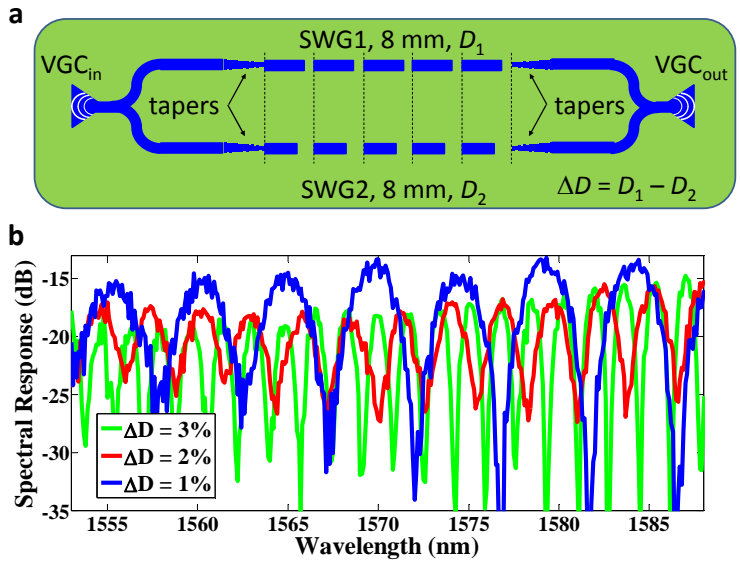

Fig. 15. (a) Schematic of MZI incorporating SWG waveguides with different duty cycles $D_{1}$ and $D_{2}$ in each arm. (b) Measured spectral responses of MZIs with $\Delta D=1 \%, 2 \%$, and $3 \%$.

While effective, length variable OTTDLs may present more complexities in terms of arranging the different waveguide lengths on-chip. One means to minimize or reduce such complexities is to develop an index variable OTTDL, whereby control of the (true) time delay is obtained by varying the group index or propagation velocity of light in the waveguides. Recently, Gasulla et al. exploited the parallelism inherent in multicore fibers to implement an index variable OTTDL [48,49]: the true time delay of each tap was controlled by designing a physical dimension or material doping concentration of each fiber core. Glesk et al. demonstrated that SWG waveguides can be used to create a delay or path mismatch in an MZI and exploited this feature to demonstrate all-optical switching and ultrafast optical sampling [50]. We expanded on these notions and used the ability of tailoring the effective index of an SWG waveguide through control of its duty cycle $D$ to realize an integrated index variable OTTDL [30].

To investigate the minimum incremental time delay that is possible, we fabricated 2-arm MZIs incorporating SWG waveguides having different duty cycles in each arm, i.e., with a duty cycle difference $\Delta D$ as shown in Fig. 15(a). Both SWG waveguides in the MZI have the same length of $8 \mathrm{~mm}$ and we designed three MZIs with $\Delta D=1 \%, 2 \%$, and $3 \%$. The SWG waveguide cross-section is $h=220 \mathrm{~nm} \times w=500 \mathrm{~nm}$ and we used $\Lambda=250 \mathrm{~nm}$. The SWG waveguides are coupled to conventional silicon strip waveguides used for the Y-branches; we use the same SWG taper designs as for the SWG BGs and MRRs described in Sections $3 \mathrm{~A}$ and $3 \mathrm{~B}$. We extract the temporal delay differences between the MZI arms by measuring the corresponding FSRs. The results are summarized in Fig. 15 (b): for $\Delta D=1 \%, 2 \%$, and $3 \%$, the FSRs are $\sim 5 \mathrm{~nm}, \sim 2.9$ $\mathrm{nm}$, and $\sim 1.7 \mathrm{~nm}$, respectively, corresponding to delay differences of $1.6 \mathrm{ps}, 2.8 \mathrm{ps}$, and $4.7 \mathrm{ps}$. This OTTDL approach can provide time delays of a few picoseconds with $8 \mathrm{~mm}$ long waveguides. The minimum achievable time delay will scale with the length of the SWG waveguide; sub-picosecond time delays can thus be expected with shorter waveguides $(<8 \mathrm{~mm})$. 
a

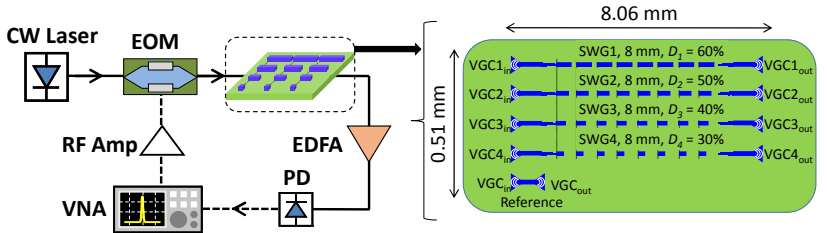

b

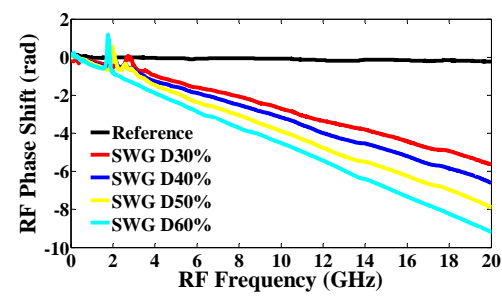

Fig. 16. (a) Schematic of OTTDL based on an array of 4 SWG waveguides and experimental setup for microwave phase shift measurements. (b) Measured RF phase shift vs. modulation frequency for an optical carrier at $1565 \mathrm{~nm}$.

To evaluate the approach for microwave phase shifting, we fabricated an OTTDL comprising 4 separate straight SWG waveguides of the same length $(8 \mathrm{~mm})$ and where the duty cycles are varied in $10 \%$ increments from $D_{1}=60 \%$ to $D_{4}=$ $30 \%$; a reference path comprising a short length of nanowire waveguide that connects the input and output VGCs is also included, see Fig. 16(a). The SWG waveguides are separated by $127 \mu \mathrm{m}$ (corresponding to the spatial separation of the VGCs and the fibers in the fiber ribbon array used for input and output coupling) and the OTTDL occupies a total chip area of $\sim 4 \mathrm{~mm}^{2}$, i.e., $0.51 \mathrm{~mm} \times 8.06 \mathrm{~mm}$. The SWG waveguides have the same parameters as for the 2-arm MZIs.

First, we measured the RF phase shift as a function of frequency from the 4 different SWG waveguides using a vector network analyzer (VNA) as illustrated in Fig. 16(a). We then calculated the time delay of each waveguide from the slope of the measured phase shift vs. frequency response. The incremental time delays are $8.9 \mathrm{ps}, 10.7 \mathrm{ps}$, and $7.9 \mathrm{ps}$ between the SWG waveguides with $D_{1}=60 \%, D_{2}=50 \%, D_{3}=40 \%$, and $D_{4}=30 \%$, respectively. Note that while the duty cycles of the SWG waveguides are varied in increments of $10 \%$, this does not translate into a linear change in incremental time delay as the group index of the SWG waveguide is not a linear function of duty cycle.

\section{SumMARY AND OUTLOOK}

Subwavelength structures have attracted considerable interest over the years and the first demonstration of an optical waveguide with an SWG metamaterial reported less than 10 years ago has fueled the development of PICs hat exploit the unique properties of SWG waveguides. The SWG waveguide 'toolbox' comprises a rich variety of components for processing light, including polarization control, power coupling, phase control (e.g., time delay), and wavelength filtering. There is a high degree of flexibility to tailor the characteristics of the SWG waveguide structure and improvements in fabrication processes will enhance performance characteristics of the various devices. The realization of low loss components, particularly waveguide crossings and bends, is perhaps the greatest appeal of SWG waveguide-based devices as these allow potentially for very dense integration of components in very compact forms. Most of the SWG waveguide-based structures demonstrated to date have been either passive, or have involved some form of thermal tuning. The next steps will likely involve the development of other active elements that do not rely on thermal mechanisms, for switching and modulation [51]. The technological developments described in this paper, along with others in SiP and SWG structures, should allow for more complex integrated systems that can provide increased functionality and performance for optical communications and MWP applications.

\section{ACKNOWLEDGMENT}

We thank R. Ashrafi, R. Adams, I. Gasulla, and J. Capmany for their contributions to the work on SWG waveguide-based ODLs.

\section{REFERENCES}

[1] R. A. Soref, "The past, present, and future of silicon photonics," IEEE J. Sel. Topics in Quantum Electron., vol. 12, no. 6, pp. 1678-1687, Nov/Dec 2006.

[2] M. Lipson, "Silicon photonics: the optical spice rack," IET Electron. Lett., vol. 45, no. 12, pp. 575-577, Jun 2009.

[3] S. Koehl, A. Liu, and M. Paniccia, "Integrated silicon photonics: harnessing the data explosion," Opt. and Photon. News, vol. 22, no. 3, pp. 24-29, Mar 2011.

[4] M. Streshinsky, M. Hochberg, P. Lo, C. Galland, T. Baehr-Jones, and A. E.-J. Lim, "The road to affordable, large-scale silicon photonics," Opt. Photon. News, vol. 24, no. 9, pp. 33-39, Sep 2013.

[5] R. Halir, A. Ortega-Moñux, J. H. Schmid, C. Alonso-Ramos, J. Lapointe, D.-X. Xu, J. G. Wangüemert-Pérez, I. Molina-Fernández, and S. Janz, "Recent advances in silicon waveguide devices using sub-wavelength gratings," IEEE J. of Sel. Topics in Quantum Electron., vol. 20, no. 4, pp. 279-291, Jul/Aug 2014.

[6] R. Halir, P. J. Bock, P. Cheben, A. Ortega-Moñux, C. Alonso-Ramos, J. H. Schmid, J. Lapointe, D.-X. Xu, J. G. Wangüemert-Pérez, Í. MolinaFernández, and S. Janz, "Waveguide sub-wavelength structures: a review of principles and applications," Lasers and Photonics Review, vol. 9, no. 1, pp. 25-49, Jan 2014.

[7] P. Cheben, R. Halir, J. H. Schmid, H. A. Atwater, and D. R. Smith, "Subwavelength integrated photonics," Nature, vol. 560, pp. 565-572, Aug 2018.

[8] S. M. Rytov, "Electromagnetic properties of a finely stratified medium," Soviet Physics JETP-USSR, vol. 2, no. 3, pp. 466-475, Mar 1956.

[9] P. Lalanne and J. P. Hugonin, "High-order effective-medium theory of subwavelength gratings in classical mounting: application to volume holograms," J. Opt. Soc. Am. A, vol. 15, no. 7, pp. 1843-1851, Jul 1998.

[10] R. Halir, P. Cheben, S. Janz, D.-X. Xu, Í. Molina-Fernández, and J. G. Wangüemert-Pérez, "Waveguide grating coupler with subwavelength microstructures," Opt. Lett., vol. 34, no. 9, pp. 1408-1410, May 2009.

[11] R. Halir, P. Cheben, J. H. Schmid, R. Ma, D. Bedard, S. Janz, D.-X. Xu, A. Densmore, J. Lapointe, and Í. Molina-Fernández, "Continuously apodized fiber-to-chip surface grating coupler with refractive index engineered subwavelength structure," Opt. Lett., vol. 35, no. 19, pp. 32433245 , Oct 2010.

[12] X. Chen, H. K. Tsang, "Polarization-independent grating couplers for silicon-on-insulator nanophotonics waveguides," Opt. Lett. vol. 36, no. 6, pp. 796-798, Mar 2011.

[13] P. J. Bock, P. Cheben, J. H. Schmid, J. Lapointe, A. Delâge, S. Janz, G. C. Aers, D.-X. Xu, A. Densmore, and T. J. Hall, "Subwavelength grating periodic structures in silicon-on-insulator: a new type of microphotonic waveguide," Opt. Express, vol. 18, no. 19, pp. 20251-20262, Sep 2010.

[14] A. Ortega-Moñux, L. Zavargo-Peche, A. Maese-Novo, Í. MolinaFernández, R. Halir, J. Wangüemert-Pérez, P. Cheben, and J. H. Schmid, "High-performance multimode interference coupler in silicon waveguides with subwavelength structures," IEEE Photon. Technol. Lett., vol. 23, no. 19, pp. 1406-1408, Oct 2011. 
[15] R. Halir, P. Cheben, J. M. Luque-González, J. D. Sarmiento-Merenguel, J. H. Schmid, G. Wangüemert-Pérez, D.-X. Xu, S. Wang, A. OrtegaMoñux, and Í. Molina-Fernández, "Ultra-broadband nanophotonic beamsplitter using an anisotropic metamaterial," Lasers and Photonics Review, vol. 10, no. 6, pp. 1039-1046, Nov 2016.

[16] P. J. Bock, P. Cheben, J. H. Schmid, A. Delâge, D.-X. Xu, S. Janz, and T. J. Hall, "Sub-wavelength grating mode transformers in silicon slab waveguides," Opt. Express, vol. 17, no. 21, pp. 19120-19133, Oct 2009.

[17] A. V. Velasco, M. L. Calvo, P. Cheben, A. Ortega-Moñux, J. H. Schmid, C. A. Ramos, İ. Molina-Fernández, J. Lapointe, M. Vachon, S. Janz, and D.-X. Xu, "Ultracompact polarization converter with a dual subwavelength trench built in a silicon-on-insulator waveguide," Opt. Lett., vol. 37, no. 3, pp. 365-367, Feb 2012.

[18] P. J. Bock, P. Cheben, J. H. Schmid, J. Lapointe, A. Delâge, D.-X. Xu, S. Janz, A. Densmore, and T. J. Hall, "Subwavelength grating crossings for silicon wire waveguides," Opt. Express, vol. 18, no. 15, pp. 16146-16155, Jul 2010.

[19] V. Donzella, A. Sherwali, J. Flueckiger, S. T. Fard, S. M. Grist, and L. Chrostowski, "Sub-wavelength grating components for integrated optics applications on SOI chips," Opt. Express, vol. 22, no. 17, pp. 2103721050 , Aug 2014.

[20] J. Wang, I. Glesk, and L. R. Chen, "Subwavelength grating filtering devices," Opt. Express, vol. 22, no. 13, pp. 15335-15345, Jun 2014.

[21] J. Wang, I. Glesk, and L. R. Chen, "Subwavelength grating devices in silicon photonics," Sci. Bull., vol. 61, no. 11, pp. 879-888, Apr 2016.

[22] J. Wang, I. Glesk, and L. R. Chen, "Subwavelength grating Bragg grating filters in silicon-on-insulator," IET Electron. Lett., vol. 51, no. 9, pp. 712713, Apr 2015

[23] J. Čtyroký, J. G. Wangüemert-Pérez, P. Kwiecien, I. Richter, J. Litvik, J. H. Schmid, Í. Molina-Fernández, A. Ortega-Moñux, M. Dado, and P. Cheben, "Design of narrowband Bragg spectral filters in subwavelength grating metamaterial waveguides," Opt. Express, vol. 26, no. 1, pp. 179194, Jan 2018.

[24] V. Donzella, A. Sherwali, J. Flueckiger, S. M. Grist, S. T. Fard, and L. Chrostowski, "Design and fabrication of SOI micro-ring resonators based on sub-wavelength grating waveguides," Opt. Express, vol. 23, no. 4, pp. 4791-4803, Feb 2015.

[25] J. Flueckiger, S. Schmidt, V. Donzella, A. Sherwali, D. M. Ratner, L. Chrostowski, and K. C. Cheung, "Sub-wavelength grating for enhanced ring resonator biosensor," Opt. Express, vol. 24, no. 14, pp. 15672-15686, Jul 2016.

[26] B. Naghdi and L. R. Chen, "Silicon photonic contradirectional couplers using subwavelength grating waveguides," Opt. Express, vol. 24, no. 20, pp. 23429-23438, Oct 2016.

[27] B. Liu, Y. Zhang, Y. He, X. Jiang, J. Peng, C. Qiu, and Y. Su, "Silicon photonic bandpass filter based on apodized subwavelength grating with high suppression ratio and short coupling length," Opt. Express, vol. 25, no. 10, pp. 11359-11364, May 2017.

[28] B. Naghdi and L. R. Chen, "Spectral engineering of subwavelength grating-based contradirectional couplers," Opt. Express, vol. 25, no. 21, pp. 25310-25317, Oct 2017.

[29] B. Naghdi and L. R. Chen, "Silicon photonic four-channel optical adddrop multiplexer enabled by subwavelength grating waveguides," IEEE Photon. J., vol. 10, no. 4, pp. 6601510, Aug 2018.

[30] J. Wang, R. Ashrafi, R. Adams, I. Glesk, I. Gasulla, J. Capmany, and L. R. Chen, "Subwavelength grating enabled on-chip ultra-compact optical true time delay line," Sci. Reports, vol. 6, 30235, Jul 2016

[31] G. Gao, M. Luo, X. Li, Y. Zhang, Q. Huang, Y. Wang, X. Xiao, Q. Yang, and J. Xia, "Transmission of $2.86 \mathrm{~Tb} / \mathrm{s}$ data stream in silicon subwavelength grating waveguides," Opt. Express, vol. 25, no. 3, pp. 2918-2927, Feb 2017.

[32] Z. Wang, X. Xu, D. Fan, Y. Wang, H. Subbaraman, and R. T. Chen, "Gemotrical tuning art for entirely subwavelength grating waveguide based integrated photonics circuits," Sci. Reports, vol. 6, 24016, May 2016.

[33] Y. Wang, X. Wang, J. Flueckiger, H. Yun, W. Shi, R. Bojko, N A. F. Jaeger, and L. Chrostowski, "Focusing sub-wavelength grating couplers with low back reflections for rapid prototyping of silicon photonic circuits," Opt. Express, vol. 22, no. 17, pp. 20652-20662, Aug 2014.

[34] T. E. Murphy, J. T. Hastings, and H. I. Smith, "Fabrication and characterization of narrow-band Bragg-reflection filters in Silicon-onInsulator ridge waveguides," IEEE/OSA J. Lightw. Technol., vol. 19, no. 12, pp. 1938-1942, Dec. 2001.

[35] X. Wang, W. Shi, H. Yun, S. Grist, N. A. F. Jaeger, and L. Chrostowski, "Narrow-band waveguide Bragg gratings on SOI wafers with CMOS- compatible fabrication process," Opt. Express, vol. 20, no. 14, pp. 15547 15558, Jul 2012.

[36] W. Shi, X. Wang, C. Lin, H. Yun, Y. Liu, T. Baehr-Jones, M. Hochberg, N. A. Jaeger, and L. Chrostowski, "Silicon photonic grating-assisted, contra-directional couplers," Opt. Express, vol. 21, no. 3, pp. 3633-3650 Feb 2013.

[37] G. Agrawal and S. Radic, "Phase shifted fiber Bragg grating and their application fo wavelength demultiplexing," IEEE Photon. Technol. Lett., vol. 6, no. 8, pp. 995-997, Aug 1994.

[38] D. Charron, J. St-Yves, A. Jafari, S. LaRochelle, and W. Shi, "Subwavelength-grating contradirectional couplers for large stopband filters," Opt. Lett., vol. 43., no. 4, pp. 895-898, Feb 2018.

[39] S. R, R. K. Gupta, N. DasGupta, and B. K. Das, "Ultra-broadband adddrop filter/switch circuit using subwavelength grating waveguides," IEEE J. Sel. Topics in Quantum Electron., vol. 25, no. 3, 8300111, May/Jun 2018.

[40] W. Shi, H. Yun, C. Lin, M. Greenberg, X. Wang, Y. Wang, S. T. Fard, J. Flueckiger, N. A. Jaeger, and L. Chrostowski, "Ultra-compact, flat-top demultiplexer using anti-reflection contra-directional couplers for CWDM networks on silicon," Opt. Express, vol. 21, no. 6, pp. 6733-6738, Mar 2013.

[41] T. Mizuochi, T. Kitayama, K. Shimizu, and K. Ito, "Interferometric crosstalk-free optical add/drop multiplexer using Mach-Zehnder-based fiber gratings," IEEE/OSA J. Lightw. Technol., vol. 16, no. 2, pp. 65-276, Feb 1998.

[42] A. E. Willner, B. Zhang, L. Zhang, L. Yan, and I. Fazal, "Optical signal processing using tunable delay elements based on slow light," IEEE J. of Sel. Topics in Quantum Electron., vol. 14, no. 3, pp. 691-705, May/Jun 2008.

[43] J. Capmany, J. Mora, I. Gasulla, J. Sancho, J. Lloret, and S. Sales, "Microwave photonic signal processing," IEEE/OSA J. Lightw. Technol., vol. 31, no. 4, pp. 571-586, Feb. 2013.

[44] W. Ng, A. A. Walston, G. L. Tangonan, J. J. Lee, I. L. Newberg, and N. Bernstein, "The first demonstration of an optically steered microwave phased array antenna using true-time-delay," IEEE/OSA J. Lightw. Technol., vol. 9, no. 9, pp. 1124-1131, Sep 1991.

[45] S. Yegnanarayanan, P. Trinh, F. Coppinger, and B. Jalali, "Compact silicon-based integrated optic time delays," IEEE Photon. Technol. Lett., vol. 9, no. 5, pp. 634-635, May 1997.

[46] A. Melloni, A. Canciamilla, C. Ferrari, F. Morichetti, L. O'Faolain, T. F. Krauss, R. De La Rue, A. Samarelli, and M. Sorel, "Tunable delay lines in silicon photonics: coupled resonators and photonic crystals: a comparison,” IEEE Photon. J., vol. 2, no. 2, pp. 181-194, Apr 2010.

[47] G. Lenz, B. J. Eggleton, C. K. Madsen, and R. E. Slusher, "Optical delay lines based on optical filters," IEEE J. Quantum Electron., vol. 37, no. 4, pp. 525-532, Apr 2001.

[48] I. Gasulla and J. Capmany, "Microwave photonics applications of multicore fibers," IEEE Photon. J., vol. 4, no. 3, pp. 877-888, Jun 2012.

[49] S. Garcia and I. Gasulla, "Design of heterogeneous multicore fibers as sampled true-time delay lines," Opt. Lett., vol. 40, no. 4, pp. 621-624, Feb 2015 .

[50] I. Glesk, P. J. Bock, P. Cheben, J. H. Schmid, J. Lapointe, and S. Janz, "All-optical switching using nonlinear subwavelength Mach-Zehnder on silicon," Opt. Express, vol. 19, pp. 14031-14039, 2011.

[51] Z. Pan, X. Xu, C.-J. Chung, H. Dalir, H. Yan, K. Chen, Y. Wang, B. Jia, and R. T. Chen, "High-speed modulator based on electro-optic polymer infiltrated subwavelength grating waveguide ring resonator," Laser Photonics Rev., vol. 12, 1700300, May 2018.

Lawrence R. Chen (S'95, SM'05) received the B.Eng. degree in electrical engineering and mathematics from McGill University, Montreal, QC, Canada, in 1995 and the M.A.Sc. and $\mathrm{Ph} . \mathrm{D}$. degrees in electrical and computer engineering in 1997 and 2000, respectively. Since 2000, he has been with the Department of Electrical and Computer Engineering at McGill University. His research interests are in optical communications, fiber and integrated optics, and microwave photonics (in particular, active and passive devices in silicon photonics for optical and microwave signal processing) as well as engineering education and teaching pedagogy. 
Dr. Chen is the former Editor-in-Chief for the IEEE Photonics Newsletter, a former Topical Editor of Optics Letters, an Editor of Optics Communications, and a Subject Editor of IET Electronics Letters. He is a Fellow of the Optical Society of America.

Junjia Wang (S'11) received the B.A.Sc. degree in electrical and computer engineering from University of Toronto, Toronto, ON, Canada, in 2009 and the M.Eng. and Ph.D. degrees in electrical and computer engineering from McGill University in 2012 and 2016, respectively. He was with Optoelectronics Research Centre at University of Southampton from mid-2015 to end-2016 under the Photonic HyperHighway project as a Research Fellow. He is now with the Department of Engineering at the University of Cambridge as a Research Associate. His research interests are in optical communications, integrated optics, and optoletectronics.

Behnam Naghdi (S'11) received the B.Sc. and M.A.Sc. degrees in electrical engineering from Sharif University of Technology, Tehran, Iran, in 2010 and 2013, respectively. Since 2014, he has been a Ph.D. student with the Department of Electrical and Computer Engineering at McGill University, Montreal, QC, Canada. His research focus is on the design and evaluation of passive and active photonic integrated devices for optical signal processing and microwave photonics applications.

Ivan Glesk (M'01, SM'01) received the Ph.D. degree in quantum electronics and optics from Comenius University, Bratislava, Slovak Republic, in 1989 and the D.Sc. degree from the Slovak Academy of Sciences. In 1986, he joined the Department of Experimental Physics, Comenius University, where he later became a Professor of Physics. From 1990, he was IREX Fellow and later Senior Research Scholar in the Department of Electrical Engineering at Princeton University, Princeton, NJ, USA. Since 2007, he has been with the Department of Electronic and Electrical Engineering at University of Strathclyde in Glasgow, UK. His current research interests include optical communications, all-optical signal processing, and integrated ultrafast optical devices for applications in optical networks. Dr. Glesk is an author and coauthor of more than 300 publications and holds five patents. He is on the International advisory board of Optica Aplicata and a recipient of the International Research and Exchanges Board (IREX) Fellowship. 